\title{
Formation Modelling and 6DOF Spacecraft Coordination Control
}

\author{
Raymond Kristiansen, Per Johan Nicklasson and Jan Tommy Gravdahl
}

\begin{abstract}
In this paper we present a 6DOF Euler-Lagrange model formulation of relative translation and rotation in a leader-follower spacecraft formation. To show the applicability of the model, we also present a solution to the problem of relative translational and rotational tracking, using the concept of vectorial integrator backstepping. The controller solution utilizes the quaternion representation to achieve a shorter rotation path on commanded attitude changes, and the equilibrium points in the closed-loop system are proved to be uniformly asymptotically stable. Finally, simulation results are presented to show the performance of the derived control law, together with the impact of orbital perturbations when these are left uncompensated.
\end{abstract}

\section{INTRODUCTION}

\section{A. Background}

Spacecraft flying in formation are revolutionizing our way of performing space-based operations, and bring out several advantages in space mission accomplishment, as well as new opportunities and applications for such missions. The concept makes the way for new and better applications in space industry, such as improved monitoring of the Earth and its surrounding atmosphere, geodesy, deep-space imaging and exploration and even in-orbit spacecraft servicing and maintenance. The replacement of traditionally large and complex spacecraft with an array of simpler microsatellites introduces a multitude of advantages regarding mission cost and performance. However, the advantages of using spacecraft formations come at a cost of increased complexity and technological challenges. Formation flying introduces a control problem with strict and time-varying boundaries on spacecraft reference trajectories, and requires detailed knowledge and tight control of relative distances and velocities for participating spacecraft.

\section{B. Previous Work}

Synchronized control of spacecraft formations in Earth orbit has received increased attention over the last years. From the first models of relative translation in spacecraft formations in circular orbits presented in [1], [2], later achievements include nonlinear models as presented in e.g. [3], [4], and later in [5] and [6], derived for arbitrary orbital eccentricity and with added terms for orbital perturbations. Models of both translational and rotational motion in a leader-follower spacecraft formation have been considered by

R. Kristiansen and P. J. Nicklasson are with the Department of Computer Science, Electrical Engineering and Space technology, Narvik University College, N-8505 Narvik, Norway

J. T. Gravdahl is with the Department of Engineering Cybernetics, Norwegian University of Science and Technology, N-7034 Trondheim, Norway few researchers, and most of the previous work has focused on translational models only. However, notable exceptions are [4], [7], where models of coupled translational and rotational motion were derived. State feedback tracking control laws for relative position and attitude were developed in [4], [8], and these solutions were proved to result in exponentially stable equilibrium points in the closed loop system. Semiglobal asymptotic convergence of relative position and attitude errors was proved in [9] for an adaptive output feedback controller using relative position only, tracing the steps of [7]. The stability result was proved using a Lyapunov framework and standard signal-chasing arguments.

\section{Contribution}

As described above, several researchers have published results on modelling and control of leader-follower spacecraft formations. As a result of visions for tighter spacecraft formations in highly elliptic orbits, the quality of the previously presented models has increased. Our contribution to the ongoing research is a 6DOF Euler-Lagrange model formulation of relative spacecraft translation and rotation (cf. [10], [11]). The motivation for a 6DOF model is scenarios where you need to control both relative translation and rotation between spacecraft in the formation. Examples are observer-type formations with viewing constraints, in-orbit surveillance operations and spacecraft rendezvous and docking. In addition, the dynamical effect of non-conservative actuators and orbital perturbations on the spacecraft in the formation is a coupling of the rotational and translational motion of the follower spacecraft.

The model is tailored for control purposes, and to show the applicability of the model, we present a state feedback solution to the problem of tracking relative translation and rotation in a leader-follower spacecraft formation, using the concept of vectorial integrator backstepping. The controller is an extension of earlier work in [12] on relative attitude control. As in the latter paper, the solution presented here also utilizes the quaternion representation to achieve a shorter rotation path on commanded attitude changes.

The rest of the paper is organized as follows: Section II defines the different reference frames used and presents the mathematical models of relative attitude dynamics and kinematics in a leader-follower spacecraft formation. The controller design is performed in Section III, and simulation results of a system with the derived controller are presented in Section IV. Concluding remarks are given in Section V. 


\section{MODELLING}

In this section, we formulate the satellite formation problem. We describe the different reference coordinate frames, together with the necessary coordinate transformations, that form the basis for our system, before we present models of the relative translation and rotation in a leader-follower spacecraft formation.

\section{A. Cartesian Coordinate Frames}

The coordinate reference frames used throughout the paper aredefined as follows:

Earth Centered Inertial (ECI) frame: This frame is denoted $\mathcal{F}_{i}$, and has its origin located in the center of the Earth. Its $z$ axis is directed along the rotation axis of the Earth towards the celestial north pole, the $x$ axis is directed towards the vernal equinox, and finally the direction of the $y$ axis completes a right handed orthogonal frame.

Leader orbit reference frame: The leader orbit frame, denoted $\mathcal{F}_{l}$, has its origin located in the center of mass of the leader spacecraft. The $\mathbf{e}_{r}$ axis in the frame is parallel to the vector $\mathbf{r}_{l}$ pointing from the center of the Earth to the spacecraft, and the $\mathbf{e}_{h}$ axis is parallel to the orbit momentum vector, which points in the orbit normal direction. The $\mathbf{e}_{\theta}$ axis completes the right-handed orthogonal frame. The basis vectors of the frame can be defined as

$$
\mathbf{e}_{r}=\frac{\mathbf{r}_{l}}{r_{l}}, \quad \mathbf{e}_{\theta}=\mathbf{e}_{h} \times \mathbf{e}_{r} \quad \text { and } \quad \mathbf{e}_{h}=\frac{\mathbf{h}}{h},
$$

where $\mathbf{h}=\mathbf{r}_{l} \times \dot{\mathbf{r}}_{l}$ is the angular momentum vector of the orbit, $h=|\mathbf{h}|$ and $r_{l}=\left|\mathbf{r}_{l}\right|$.

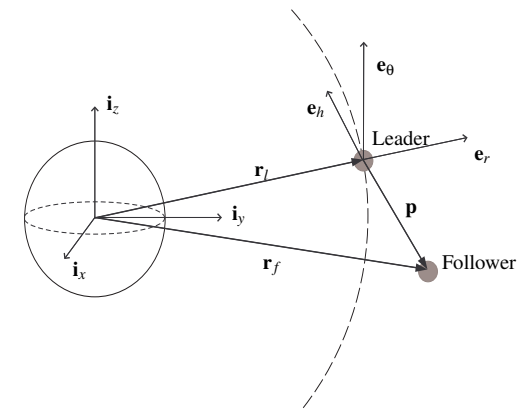

Fig. 1. Reference coordinate frames [13].

Follower orbit reference frame: This frame has its origin in the center of mass of the follower spacecraft, and is denoted $\mathcal{F}_{f}$. The vector pointing from the center of the Earth to the center of the follower orbit frame is denoted $\mathbf{r}_{f}$. Its origin

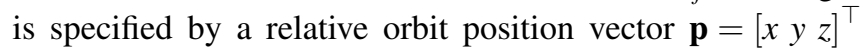
expressed in $\mathcal{F}_{l}$ frame components, and the frame unit vectors align with the basis vectors of $\mathcal{F}_{l}$. Accordingly,

$$
\mathbf{p}=\mathbf{r}_{f}-\mathbf{r}_{l}=x \mathbf{e}_{r}+y \mathbf{e}_{\theta}+z \mathbf{e}_{h} .
$$

Body reference frames: For both the leader and the follower spacecraft, body reference frames are defined and denoted $\mathcal{F}_{b l}$ and $\mathcal{F}_{b f}$, respectively. These frames have, similar to the orbit frame, the origin located in the center of mass of the respective spacecraft, but the basis vectors are fixed in the spacecraft body and coincide with its principal axis of inertia.

\section{B. Coordinate Frame Transformations}

1) Rotation from ECI to Leader Orbit Frame: The rotation from the ECI frame to the leader orbit frame is dependent on the parameters of the leader spacecraft orbit, and can be expressed by three consecutive rotations. The total rotation matrix can be written

$$
\mathbf{R}_{i}^{l}=\mathbf{R}_{z, \omega+v} \mathbf{R}_{x, i} \mathbf{R}_{z, \Omega}
$$

where $\Omega$ is the right ascension of the ascending node of the orbit, $i$ is the inclination of the orbit, $v$ is the true anomaly of the leader orbit, and $\omega$ is the argument of perigee of the same. The sum of $v$ and $\omega$ represents the location of the spacecraft relative to the ascending node.

2) Body Frame Rotation: The rotation matrix describing rotations from an orbit frame to a body frame can be described by

$$
\mathbf{R}_{o}^{b}=\left[\begin{array}{lll}
\mathbf{c}_{1} & \mathbf{c}_{2} & \mathbf{c}_{3}
\end{array}\right]=\mathbf{I}+2 \eta \mathbf{S}(\varepsilon)+2 \mathbf{S}^{2}(\varepsilon)
$$

where the elements $\mathbf{c}_{i}$ are directional cosines, and $\mathbf{q}=$ $\left[\eta, \varepsilon^{\top}\right]^{\top}$ are the Euler parameters, which satisfy the constraint

$$
\eta^{2}+\varepsilon^{\top} \varepsilon=1 .
$$

The matrix $\mathbf{S}(\cdot)$ is the cross product operator given by

$$
\mathbf{S}(\varepsilon)=\boldsymbol{\varepsilon} \times=\left[\begin{array}{ccc}
0 & -\varepsilon_{z} & \varepsilon_{y} \\
\varepsilon_{z} & 0 & -\varepsilon_{x} \\
-\varepsilon_{y} & \varepsilon_{x} & 0
\end{array}\right]
$$

when $\varepsilon=\left[\begin{array}{lll}\varepsilon_{x} & \varepsilon_{y} & \varepsilon_{z}\end{array}\right]^{\top}$. The inverse rotation is given by the complex conjugate of $\mathbf{q}$ as $\overline{\mathbf{q}}=\left[\eta,-\varepsilon^{\top}\right]^{\top}$.

\section{Relative Translation}

The fundamental differential equation of the ideal twobody problem can be expressed as [14]

$$
\frac{d^{2} \mathbf{r}}{d t^{2}}+\frac{\mu}{r^{3}} \mathbf{r}=\mathbf{0}
$$

where $\mathbf{r}=\mathbf{r}_{2}-\mathbf{r}_{1}$ is the relative position of masses $m_{1}$ and $m_{2}$ with respective ECI position vectors $\mathbf{r}_{1}$ and $\mathbf{r}_{2}$, and $\mu=$ $G\left(m_{1}+m_{2}\right)$ with $G$ as the universal constant of gravity. This equation can be generalized to include force terms due to orbital perturbations and control input vectors from onboard actuators. Accordingly, (7) can be expressed as

$$
\ddot{\mathbf{r}}_{s}=-\frac{\mu}{r_{s}^{3}} \mathbf{r}_{s}+\frac{\mathbf{f}_{d s}}{m_{s}}+\frac{\mathbf{f}_{a s}}{m_{s}}
$$

where $\mathbf{f}_{d s} \in \mathbb{R}^{3}$ is the disturbance term due to external effects and $\mathbf{f}_{a s} \in \mathbb{R}^{3}$ is the actuator force. The superscript/subscript $s$ is used in general to denote the spacecraft in question, so $s=l, f$ for the leader and follower spacecraft, respectively. In addition, spacecraft masses are assumed to be small relative to the mass of the Earth $M_{e}$, so $\mu \approx G M_{e}$. By using the true anomaly $v$ of the leader spacecraft, and denoting relative 
velocity as $\mathbf{v}=\dot{\mathbf{p}}$, we obtain the nonlinear position dynamics represented in the $\mathcal{F}_{l}$ frame on the form (cf. [6],[15])

$$
m_{f} \dot{\mathbf{v}}+\mathbf{C}_{t}(\dot{\mathrm{v}}) \mathbf{v}+\mathbf{D}_{t}\left(\dot{\mathrm{v}}, \ddot{\mathrm{v}}, r_{f}\right) \mathbf{p}+\mathbf{n}_{t}\left(r_{l}, r_{f}\right)=\mathbf{F}_{a}+\mathbf{F}_{d}
$$

where

$$
\mathbf{C}_{t}(\dot{\mathrm{v}})=2 m_{f} \dot{\mathrm{v}}\left[\begin{array}{ccc}
0 & -1 & 0 \\
1 & 0 & 0 \\
0 & 0 & 0
\end{array}\right] \in S S(3)
$$

is a skew-symmetric Coriolis-like matrix,

$$
\mathbf{D}_{t}\left(\dot{\mathrm{v}}, \ddot{\mathrm{v}}, r_{f}\right) \mathbf{p}=m_{f}\left[\begin{array}{ccc}
\frac{\mu}{r_{f}^{3}}-\dot{\mathrm{v}}^{2} & -\ddot{\mathrm{v}} & 0 \\
\ddot{\mathrm{v}} & \frac{\mu}{r_{f}^{3}}-\dot{\mathrm{v}}^{2} & 0 \\
0 & 0 & \frac{\mu}{r_{f}^{3}}
\end{array}\right] \mathbf{p}
$$

may be viewed as a time-varying potential force, and

$$
\mathbf{n}_{t}\left(r_{l}, r_{f}\right)=m_{f} \mu\left[\frac{r_{l}}{r_{f}^{3}}-\frac{1}{r_{l}^{2}} \quad 0 \quad 0\right]^{\top} .
$$

The composite disturbance force $\mathbf{F}_{d}$ and the relative control force $\mathbf{F}_{a}$ are given by

$$
\mathbf{F}_{d}=\mathbf{f}_{d f}-\frac{m_{f}}{m_{l}} \mathbf{f}_{d l} \quad \mathbf{F}_{a}=\mathbf{f}_{a f}-\frac{m_{f}}{m_{l}} \mathbf{f}_{a l} .
$$

\section{Relative Rotation}

The time derivative of a matrix $\mathbf{R}_{b}^{a}$ as in (4) can according to [11] be written as

$$
\dot{\mathbf{R}}_{b}^{a}=\mathbf{S}\left(\omega_{a, b}^{a}\right) \mathbf{R}_{b}^{a}=\mathbf{R}_{b}^{a} \mathbf{S}\left(\omega_{a, b}^{b}\right)
$$

where $\omega_{a, b}^{b}$ is the angular velocity of frame $b$ relative to frame $a$ represented in frame $b$ and $\mathbf{S}(\cdot)$ is the cross product operator described in (6). The kinematic differential equations for a spacecraft in its orbit frame can be found from (14) together with (4) as

$$
\dot{\mathbf{q}}_{s}=\mathbf{T}\left(\mathbf{q}_{s}\right) \omega_{s, s b}^{s b}, \quad \mathbf{T}\left(\mathbf{q}_{s}\right)=\frac{1}{2}\left[\begin{array}{c}
-\varepsilon_{s}^{T} \\
\eta_{s} \mathbf{I}+\mathbf{S}\left(\varepsilon_{s}\right)
\end{array}\right]
$$

where $\omega_{s, s b}^{s b}$ is the angular velocity of the spacecraft body frame relative to the orbit frame, referenced in the body frame. Moreover, with the assumptions of rigid body movement, the dynamical model of a spacecraft can be found from Euler's momentum equation as [16]

$$
\begin{aligned}
\mathbf{J}_{s} \dot{\omega}_{i, s b}^{s b} & =-\mathbf{S}\left(\omega_{i, s b}^{s b}\right) \mathbf{J}_{s} \omega_{i, s b}^{s b}+\tau_{d s}^{s b}+\tau_{a s}^{s b} \\
\omega_{s, s b}^{s b} & =\omega_{i, s b}^{s b}+\omega_{o} \mathbf{c}_{2}
\end{aligned}
$$

where $\mathbf{J}_{s}$ is the spacecraft inertia matrix and $\omega_{i, s b}^{s b}$ is the angular velocity of the spacecraft body frame relative to the inertial frame, expressed in the body frame. The parameter $\omega_{o}$ is the orbit angular velocity, $\tau_{d}^{s b}$ is the disturbance torque, $\tau_{a}^{s b}$ is the actuator torque, and $\mathbf{c}_{2}$ is the directional cosine vector from (4).

Further, by expressing the relations in (15) and (16)-(17) for both the leader and the follower spacecraft, and utilising the quaternion product defined in [11] as

$$
\mathbf{q}=\mathbf{q}_{f} \otimes \overline{\mathbf{q}}_{l} \triangleq\left[\begin{array}{c}
\eta_{f} \eta_{l}+\varepsilon_{f}^{T} \varepsilon_{l} \\
\eta_{l} \varepsilon_{f}-\eta_{f} \varepsilon_{l}-\mathbf{S}\left(\varepsilon_{f}\right) \varepsilon_{l}
\end{array}\right]
$$

the relative attitude kinematics can be expressed as [17]

$$
\dot{\mathbf{q}}=\left[\begin{array}{c}
\dot{\eta} \\
\dot{\varepsilon}
\end{array}\right]=\mathbf{T}(\mathbf{q}) \omega, \quad \omega=\omega_{i, f b}^{f b}-\mathbf{R}_{l b}^{f b} \omega_{i, l b}^{l b}
$$

where $\omega$ is the relative angular velocity between the leader body reference frame and the follower body reference frame. Moreover, from (19) the relative attitude dynamics can be expressed as

$$
\begin{aligned}
\mathbf{J}_{f} \dot{\boldsymbol{\omega}} & =\mathbf{J}_{f} \dot{\omega}_{i, f b}^{f b}-\mathbf{J}_{f} \dot{\mathbf{R}}_{l b}^{f b} \omega_{i, l b}^{l b}-\mathbf{J}_{f} \mathbf{R}_{l b}^{f b} \dot{\omega}_{i, l b}^{l b} \\
& =\mathbf{J}_{f} \dot{\omega}_{i, f b}^{f b}-\mathbf{J}_{f} \mathbf{S}\left(\omega_{i, l b}^{f b}\right) \omega-\mathbf{J}_{f} \mathbf{R}_{l b}^{f b} \dot{\omega}_{i, l b}^{l b}
\end{aligned}
$$

where (14) and the facts that $\omega_{l b, f b}^{f b}=\omega$ and $\mathbf{S}(\mathbf{a}) \mathbf{b}=$ $-\mathbf{S}(\mathbf{b}) \mathbf{a}$ have been used. Insertion of (16), evaluated for both the leader and follower, into (20) results in ( $c f$. [15])

$$
\mathbf{J}_{f} \dot{\omega}+\mathbf{C}_{r}(\omega) \omega+\mathbf{n}_{r}(\omega)=\Upsilon_{d}+\Upsilon_{a}
$$

where

$$
\begin{aligned}
\mathbf{C}_{r}(\omega)=\mathbf{J}_{f} \mathbf{S}\left(\mathbf{R}_{l b}^{f b} \omega_{i, l b}^{l b}\right) & +\mathbf{S}\left(\mathbf{R}_{l b}^{f b} \omega_{i, l b}^{l b}\right) \mathbf{J}_{f} \\
& -\mathbf{S}\left(\mathbf{J}_{f}\left(\omega+\mathbf{R}_{l b}^{f b} \omega_{i, l b}^{l b}\right)\right)
\end{aligned}
$$

is a skew-symmetric matrix, $\mathbf{C}_{r}(\omega) \in S S(3)$,

$$
\begin{aligned}
\mathbf{n}_{r}(\omega)=\mathbf{S}\left(\mathbf{R}_{l b}^{f b} \omega_{i, l b}^{l b}\right) & \mathbf{J}_{f} \mathbf{R}_{l b}^{f b} \omega_{i, l b}^{l b} \\
& -\mathbf{J}_{f} \mathbf{R}_{l b}^{f b} \mathbf{J}_{l}^{-1} \mathbf{S}\left(\omega_{i, l b}^{l b}\right) \mathbf{J}_{l} \omega_{i, l b}^{l b}
\end{aligned}
$$

is a nonlinear term, and

$$
\Upsilon_{d}=\tau_{d f}^{f b}-\mathbf{J}_{f} \mathbf{R}_{l b}^{f b} \mathbf{J}_{l}^{-1} \tau_{d l}^{l b}, \quad \Upsilon_{a}=\tau_{a f}^{f b}-\mathbf{J}_{f} \mathbf{R}_{l b}^{f b} \mathbf{J}_{l}^{-1} \tau_{a l}^{l b}
$$

are the relative disturbance torques and relative actuator torques, respectively.

\section{E. Total Model}

To write the total 6DOF model of relative translation and rotation in the spacecraft formation, define the state vectors

$$
\mathbf{x}_{1}=\left[\begin{array}{ll}
\mathbf{p}^{\top} & \mathbf{q}^{\top}
\end{array}\right]^{\top} \quad \text { and } \quad \mathbf{x}_{2}=\left[\begin{array}{ll}
\mathbf{v}^{\top} & \omega^{\top}
\end{array}\right]^{\top} .
$$

Based on (9) and (21), the total model of the relative translational and rotational motion between the leader and the follower spacecraft can now be expressed

$$
\begin{gathered}
\quad \dot{\mathbf{x}}_{1}=\Lambda\left(\mathbf{x}_{1}\right) \mathbf{x}_{2} \\
\mathbf{M}_{f} \dot{\mathbf{x}}_{2}+\mathbf{C}(\dot{\mathrm{v}}, \omega) \mathbf{x}_{2}+\mathbf{D}\left(\dot{\mathrm{v}}, \ddot{v}, r_{f}\right) \mathbf{x}_{1}+\mathbf{n}\left(\omega, r_{l}, r_{f}\right)=\mathbf{U}+\mathbf{W}
\end{gathered}
$$

where

$$
\mathbf{M}_{f}=\left[\begin{array}{cc}
m_{f} \mathbf{I} & \mathbf{0} \\
\mathbf{0} & \mathbf{J}_{f}
\end{array}\right]
$$

is a symmetric positive definite matrix containing the mass and moments of inertia of the follower spacecraft,

$$
\Lambda\left(\mathbf{x}_{1}\right)=\left[\begin{array}{cc}
\mathbf{I} & \mathbf{0} \\
\mathbf{0} & \mathbf{T}(\mathbf{q})
\end{array}\right]
$$

is the coupling term between the first and second order dynamics,

$$
\mathbf{C}(\dot{v}, \omega)=\left[\begin{array}{cc}
\mathbf{C}_{t}(\dot{\mathrm{v}}) & \mathbf{0} \\
\mathbf{0} & \mathbf{C}_{r}(\omega)
\end{array}\right] \in S S(6)
$$


is the skew-symmetric Coriolis-like matrix,

$$
\mathbf{D}\left(\dot{\mathrm{v}}, \ddot{\mathrm{v}}, r_{f}\right) \mathbf{x}_{1}=\left[\begin{array}{cc}
\mathbf{D}_{t}\left(\dot{\mathrm{v}}, \ddot{\mathrm{v}}, r_{f}\right) & \mathbf{0} \\
\mathbf{0} & \mathbf{0}
\end{array}\right] \mathbf{x}_{1}
$$

is the time-varying potential force term,

$$
\mathbf{n}\left(\omega, r_{l}, r_{f}\right)=\left[\begin{array}{c}
\mathbf{n}_{t}\left(r_{l}, r_{f}\right) \\
\mathbf{n}_{r}(\omega)
\end{array}\right]
$$

is the composite nonlinear term, and finally

$$
\mathbf{U}=\left[\begin{array}{l}
\mathbf{F}_{a} \\
\Upsilon_{a}
\end{array}\right] \quad \text { and } \quad \mathbf{W}=\left[\begin{array}{l}
\mathbf{F}_{d} \\
\Upsilon_{d}
\end{array}\right]
$$

contains the relative input forces and orbital perturbations, respectively. The dynamical effect of orbital perturbations on the spacecraft in the formation is a coupling of the rotational and translational motion of the follower spacecraft. Perturbing forces working on the follower are dependent on its position, and especially its altitude. The resulting torques due to these perturbing forces, which cause rotational motion of the spacecraft, will thus also depend on the position. Accordingly, the rotational motion is affected by the translational motion. Similar, the effect of perturbing forces due to atmospheric drag and solar radiation on the translational motion is dependent on the orientation of the spacecraft, varying with the size of the equivalent surface area in the force direction. Therefore, the translational motion of the follower spacecraft is affected by the rotational motion of the spacecraft. The result of orbital perturbations is thus a two way coupling in the model presented in (27). Also, use of non-conservative actuators as thrusters, magnetic rods and solar sails will in some actuator configurations cause both rotational and translational spacecraft motion. For a more detailed derivation of the 6DOF model, see [15].

\section{CONTROLLER DESIGN}

Having established the 6DOF mathematical model of relative translation and rotation in a leader-follower formation, we now present a solution to the control problem. Equation (27) can be interpreted as a "mass-damper-spring" system with an added nonlinear term, and the model has many similarities with models of systems such as robot manipulators, marine crafts and underwater vehicles (cf. [10], [18]). This is convenient since automatic control theory for these types of systems is highly developed.

\section{A. Integrator Backstepping}

The control problem is to design a controller that makes the state $\mathbf{x}_{1}$ converge to and proceed to track a timevarying smooth trajectory $\mathbf{x}_{d}(t)$. The desired trajectory can be specified as

$$
\mathbf{x}_{d 1}=\left[\begin{array}{c}
\mathbf{p}_{d} \\
\mathbf{q}_{d}
\end{array}\right] \quad \mathbf{x}_{d 2}=\left[\begin{array}{c}
\mathbf{v}_{d} \\
\omega_{d}
\end{array}\right]
$$

so that

$$
\dot{\mathbf{x}}_{d 1}=\Lambda\left(\mathbf{x}_{d 1}\right) \mathbf{x}_{d 2}
$$

Following the concept of the integrator backstepping design, we define our first backstepping variable as

$$
\mathbf{z}_{1}=\left[\begin{array}{c}
\tilde{\mathbf{p}} \\
1-|\tilde{\eta}| \\
\tilde{\varepsilon}
\end{array}\right]
$$

where $\tilde{\mathbf{p}}=\mathbf{p}-\mathbf{p}_{d}$ is the translation error, and the rotation error $\tilde{\mathbf{q}}=\left[\tilde{\eta} \tilde{\varepsilon}^{\top}\right]^{\top}$ is given from the quaternion product

$$
\tilde{\mathbf{q}}=\mathbf{q} \otimes \overline{\mathbf{q}}_{d}=\left[\begin{array}{c}
\eta \eta_{d}+\varepsilon^{\top} \varepsilon_{d} \\
\eta_{d} \varepsilon-\eta \varepsilon_{d}-\mathbf{S}(\varepsilon) \varepsilon_{d}
\end{array}\right]
$$

Perfect trajectory tracking can be expressed as

$$
\mathbf{x}_{1}(t)=\mathbf{x}_{d 1}(t) \quad \Leftrightarrow \quad \tilde{\mathbf{x}}_{1}(t)=\left[\begin{array}{c}
\tilde{\mathbf{p}} \\
\tilde{\mathbf{q}}
\end{array}\right]=\left[\begin{array}{c}
\mathbf{0} \\
\pm 1 \\
\mathbf{0}
\end{array}\right]
$$

for all $t \geq 0$. Following the notation in (15), it can be shown that

$$
\dot{\tilde{\mathbf{q}}}=\mathbf{T}(\tilde{\mathbf{q}}) \tilde{\omega}=\frac{1}{2}\left[\begin{array}{c}
-\tilde{\varepsilon}^{\top} \\
\tilde{\eta} \mathbf{I}+\mathbf{S}(\tilde{\varepsilon})
\end{array}\right] \tilde{\omega}
$$

with $\tilde{\omega}=\omega-\omega_{d}$. Accordingly, the dynamics of the $\mathbf{z}_{1}$-system can be expressed as

$$
\dot{\mathbf{z}}_{1}=\mathbf{G}^{\top}\left(\tilde{\mathbf{x}}_{1}\right) \tilde{\mathbf{x}}_{2}
$$

where

$$
\mathbf{G}^{\top}\left(\tilde{\mathbf{x}}_{1}\right)=\left[\begin{array}{cc}
\mathbf{I} & \mathbf{0} \\
\mathbf{0} & \frac{1}{2}\left[\begin{array}{c}
\operatorname{sgn}(\tilde{\boldsymbol{\eta}}) \tilde{\varepsilon}^{\top} \\
(\tilde{\boldsymbol{\eta}} \mathbf{I}+\mathbf{S}(\tilde{\boldsymbol{\varepsilon}}))
\end{array}\right]
\end{array}\right]
$$

and $\tilde{\mathbf{x}}_{2}=[\tilde{\mathbf{v}}, \tilde{\omega}]$. A virtual control input is now defined as

$$
\tilde{\mathbf{x}}_{2}=\alpha_{1}+\mathbf{z}_{2}
$$

where $\alpha_{1}$ is a stabilizing function and $\mathbf{z}_{2}$ is a new state variable; Hence,

$$
\dot{\mathbf{z}}_{1}=\mathbf{G}^{\top}\left(\tilde{\mathbf{x}}_{1}\right)\left(\alpha_{1}+\mathbf{z}_{2}\right)
$$

It can be shown that

$$
\mathbf{G}\left(\tilde{\mathbf{x}}_{1}\right) \mathbf{z}_{1}=\mathbf{0} \Leftrightarrow\left[\begin{array}{c}
\tilde{\mathbf{p}} \\
\operatorname{sgn}(\tilde{\eta}) \tilde{\varepsilon}
\end{array}\right]=\mathbf{0}
$$

and the signum function $\operatorname{sgn}(\mathrm{x})$ is therefore defined nonzero as

$$
\operatorname{sgn}(x)=\left\{\begin{aligned}
-1, & x<0 \\
1, & x \geq 0
\end{aligned}\right.
$$

to avoid a singularity when $\tilde{\eta}=0$. A Lyapunov Function Candidate (LFC) is chosen as

$$
\begin{aligned}
& V_{1}=\frac{1}{2} \mathbf{z}_{1}^{\top} \mathbf{z}_{1} \\
& \dot{V}_{1}=\mathbf{z}_{1}^{\top} \dot{\mathbf{z}}_{1}=\mathbf{z}_{1}^{\top} \mathbf{G}^{\top}\left(\tilde{\mathbf{x}}_{1}\right)\left(\alpha_{1}+\mathbf{z}_{2}\right) .
\end{aligned}
$$

Furthermore, the stabilizing function $\alpha_{1}$ is chosen as

$$
\alpha_{1}=-\mathbf{K}_{1} \mathbf{G}\left(\tilde{\mathbf{x}}_{1}\right) \mathbf{z}_{1}
$$


where $\mathbf{K}_{1}=\mathbf{K}_{1}^{\top}>0$ is a feedback gain matrix. Inserting this into the LFC in (47) yields

$$
\dot{V}_{1}=-\mathbf{z}_{1}^{\top} \mathbf{G}^{\top} \mathbf{K}_{1} \mathbf{G} \mathbf{z}_{1}+\mathbf{z}_{1}^{\top} \mathbf{G}^{\top} \mathbf{z}_{2}
$$

where the argument of the matrix $\mathbf{G}\left(\tilde{\mathbf{x}}_{1}\right)$ is left out for readability. It should be noted that $\mathbf{G}^{\top} \mathbf{K}_{1} \mathbf{G}$ is a symmetric positive semidefinite matrix. The $\mathbf{z}_{1}$-system from (40) can be written accordingly as

$$
\begin{aligned}
\dot{\mathbf{z}}_{1} & =\mathbf{G}^{\top}\left(\alpha_{1}+\mathbf{z}_{2}\right) \\
& =-\mathbf{G}^{\top} \mathbf{K}_{1} \mathbf{G} \mathbf{z}_{1}+\mathbf{G}^{\top} \mathbf{z}_{2} .
\end{aligned}
$$

For the second step, (42) is differentiated to express the $\mathbf{z}_{2}$ dynamics as

$$
\begin{aligned}
\dot{\mathbf{z}}_{2} & =\dot{\tilde{\mathbf{x}}}_{2}-\dot{\alpha}_{1} \\
& =\dot{\mathbf{x}}_{2}-\dot{\mathbf{x}}_{d 2}-\dot{\alpha}_{1}
\end{aligned}
$$

and insertion of (27), with matrix arguments ignored for readability, leaves

$$
\begin{aligned}
\mathbf{M}_{f} \dot{\mathbf{z}}_{2} & =\mathbf{M}_{f} \dot{\mathbf{x}}_{2}-\mathbf{M}_{f} \dot{\mathbf{x}}_{d 2}-\mathbf{M}_{f} \dot{\alpha}_{1} \\
& =\mathbf{U}+\mathbf{W}-\mathbf{C} \mathbf{x}_{2}-\mathbf{D} \mathbf{x}_{1}-\mathbf{n}-\mathbf{M}_{f} \dot{\mathbf{x}}_{d 2}-\mathbf{M}_{f} \dot{\alpha}_{1} .
\end{aligned}
$$

A second LFC can now be expressed as

$$
V_{2}=V_{1}+\frac{1}{2} \mathbf{z}_{2}^{\top} \mathbf{M}_{f} \mathbf{z}_{2}
$$

and hence

$$
\begin{aligned}
\dot{V}_{2} & =\dot{V}_{1}+\mathbf{z}_{2}^{\top} \mathbf{J}_{f} \dot{\mathbf{z}}_{2} \\
& =\dot{V}_{1}+\mathbf{z}_{2}^{\top}\left[\mathbf{U}+\mathbf{W}-\mathbf{C} \mathbf{x}_{2}-\mathbf{D} \mathbf{x}_{1}-\mathbf{n}-\mathbf{M}_{f} \dot{\mathbf{x}}_{d 2}-\mathbf{M}_{f} \dot{\alpha}_{1}\right]
\end{aligned}
$$

Assume that the leader spacecraft is perfectly controlled in its orbit, so that $\tau_{a l}^{l b}=-\tau_{d l}^{l b}$ and $\mathbf{f}_{a l}=-\mathbf{f}_{d l}$. Assume also that the disturbance vectors $\tau_{d f}^{f b}$ and $\mathbf{f}_{d f}$ can be measured or estimated. Then, choosing the actuator torque $\mathbf{u}_{f}=$ $\left[\mathbf{f}_{a f}^{\top},\left(\tau_{a f}^{f b}\right)^{\top}\right]^{\top}$ as

$$
\begin{aligned}
\mathbf{u}_{f}= & -\mathbf{K}_{2} \mathbf{z}_{2}-\mathbf{G} \mathbf{z}_{1}-\mathbf{w}_{f}+\mathbf{C} \mathbf{x}_{2}+\mathbf{D} \mathbf{x}_{1}+\mathbf{n} \\
& +\mathbf{M}_{f} \dot{\mathbf{x}}_{d 2}+\mathbf{M}_{f} \dot{\alpha}_{1}
\end{aligned}
$$

where $\mathbf{K}_{2}=\mathbf{K}_{2}^{\top}>0$ is the feedback gain matrix for the $\mathbf{z}_{2}$ system and $\mathbf{w}_{f}=\left[\mathbf{f}_{d f}^{\top},\left(\tau_{d f}^{f b}\right)^{\top}\right]^{\top}$, leaves the LFC as

$$
\begin{aligned}
\dot{V}_{2} & =\dot{V}_{1}+\mathbf{z}_{2}^{\top}\left[-\mathbf{K}_{2} \mathbf{z}_{2}-\mathbf{G} \mathbf{z}_{1}\right] \\
& =-\mathbf{z}_{1}^{\top} \mathbf{G}^{\top} \mathbf{K}_{1} \mathbf{G} \mathbf{z}_{1}-\mathbf{z}_{2}^{\top} \mathbf{K}_{2} \mathbf{z}_{2} \\
& =-W\left(\mathbf{z}_{1}, \mathbf{z}_{2}\right) \leq \mathbf{0}
\end{aligned}
$$

and the closed-loop dynamics as

$$
\begin{aligned}
\dot{\mathbf{z}}_{1} & =-\mathbf{G}^{\top} \mathbf{K}_{1} \mathbf{G} \mathbf{z}_{1}+\mathbf{G}^{\top} \mathbf{z}_{2} \\
\mathbf{M}_{f} \dot{\mathbf{z}}_{2} & =-\mathbf{K}_{2} \mathbf{z}_{2}-\mathbf{G} \mathbf{z}_{1} .
\end{aligned}
$$

\section{B. Stability Properties}

The stability properties of the closed loop system given by (63)-(64) follows from (56) and (62). From (56) it is seen that $V_{2}\left(\mathbf{z}_{1}, \mathbf{z}_{2}\right)>0, V_{2}(0)=0$ and $V_{2}\left(\mathbf{z}_{1}, \mathbf{z}_{2}\right) \rightarrow \infty$ as $\left(\mathbf{z}_{1}, \mathbf{z}_{2}\right) \rightarrow \infty$. Similarly, by (5) and (44) it can be shown that $W\left(\mathbf{z}_{1}, \mathbf{z}_{2}\right)>0$ and $W(0)=0$, and hence $W\left(\mathbf{z}_{1}, \mathbf{z}_{2}\right)$ is positive definite. From invoking standard Lyapunov theorems ( $c f$. [19]), it follows that both of the equilibrium points $\tilde{\mathbf{x}}_{1}=\left[\begin{array}{lll}\mathbf{0}^{\top} \pm 1 & \mathbf{0}^{\top}\end{array}\right]^{\top}$ are uniformly asymptotically stable (UAS), and it follows from (38) that $\mathbf{x}_{1}(t) \rightarrow \mathbf{x}_{d 1}(t)$ as $t \rightarrow \infty$. Also, (42) and (48) implies that $\tilde{\mathbf{x}}_{2}(t) \rightarrow \mathbf{0}$ as $t \rightarrow \infty$.

Remark 1: If the first backstepping variable in (36) is chosen as

$$
\mathbf{z}_{1}=\left[\begin{array}{c}
\tilde{\mathbf{p}} \\
1-\tilde{\eta} \\
\tilde{\varepsilon}
\end{array}\right]
$$

the equilibrium point $\tilde{\mathbf{x}}_{1}=\left[\begin{array}{lll}\mathbf{0}^{\top} & 1 & \mathbf{0}^{\top}\end{array}\right]^{\top}$ will be UAS, while $\tilde{\mathbf{x}}_{1}=\left[\begin{array}{lll}\mathbf{0}^{\top}-1 \mathbf{0}^{\top}\end{array}\right]^{\top}$ will be unstable. Hence, the scalar part of the quaternion must always be regulated to the stable point where $\tilde{\eta}=1$, even if a rotation to the point where $\tilde{\eta}=-1$ requires less power. A discussion concerning this can be found in [20]. When both equilibrium points are UAS, the relative attitude can be regulated to the closest equilibrium point with respect to rotation path, which will imply less use of energy.

Remark 2: We stress that the asymptotic stability properties for the equilibrium points are only local. Since the unit quaternion parameter set is redundant, a given physical attitude for a rigid body will have two mathematical representations, where one of these includes a rotation of $2 \pi$ about an axis relative to the other. This is the advantage that is exploited by our choice of backstepping variables, to render both equilibrium points UAS, as mentioned in Remark 1. Based on the physical nature of the problem, it might be appealing to think that the equilibrium point set $\tilde{\mathbf{x}}_{1}=\left[\mathbf{0}^{\top} \pm 1 \mathbf{0}^{\top}\right]^{\top}$ has global stability properties, but this is however not the case. A discussion concerning this point can be found in [21]. It is however noted that the lack of global properties is a result of the attitude representation, and if the same controller was derived for position tracking only, the closed-loop system would have only one UGAS equilibrium point.

Remark 3: The asymptotic stability property of the equilibrium points is a result of the assumption of known disturbance vectors $\tau_{d f}^{f b}$ and $\mathbf{f}_{d f}$, and a perfectly controlled leader. If this assumption is relaxed to unknown, but bounded, disturbance vectors and leader motion, it can be shown that the stability property will degenerate to uniform practical asymptotic stability ( $c f$. [22]). That is, the state errors in the closed-loop system can be proved to converge from any initial conditions to a ball in close vicinity of the origin in a stable way, and this ball can be diminished arbitrarily by increasing the gains in the control law.

Remark 4: Notably, an alternative choice of actuator input 
is

$$
\begin{aligned}
\mathbf{u}_{f}= & -\mathbf{K}_{2} \mathbf{z}_{2}-\mathbf{G} \mathbf{z}_{1}-\mathbf{w}_{f}+\mathbf{C}\left(\mathbf{x}_{d 2}+\alpha_{1}\right)+\mathbf{D} \mathbf{x}_{1}+\mathbf{n} \\
& +\mathbf{M}_{f} \dot{\mathbf{x}}_{d 2}+\mathbf{M}_{f} \dot{\alpha}_{1}
\end{aligned}
$$

which also yields the result (62). This is due to the fifth term $\mathbf{C x}_{2}$ in (59), which is added to remove the corresponding term in (58). In (66) the term $\mathbf{C}\left(\mathbf{x}_{d 2}+\alpha_{1}\right)$ is used instead, to add up with $-\mathbf{C} \mathbf{x}_{2}$ so that

$$
\mathbf{z}_{2}^{\top}\left[\mathbf{C}\left(\mathbf{x}_{d 2}+\alpha_{1}\right)-\mathbf{C x}_{2}\right]=-\mathbf{z}_{2}^{\top} \mathbf{C} \mathbf{z}_{2}=0
$$

since the matrix $C$ is skew-symmetric.

Remark 5: Note that, besides reducing the length of the rotation path, no attempt is made to minimize the fuel consumption during the maneuver. The main point in this paper is to show that standard controller design methods developed for general nonlinear time-varying systems, and specifically Euler-Lagrange systems, are straightforward to use on our model formulation, and topics such as fuel minimization/equalization, optimal rotation etc. are therefore topics for further research.

\section{Implementation}

The control law given by (59) contains the expression $\dot{\alpha}_{1}$ that involves time derivatives of the states, and this should be avoided when the control law is implemented. A possible solution is to use the model equations as a sort of smooth differentiators, instead of differentiating the measurements directly. The time differentiation of (48) can be performed as

$$
\dot{\alpha}_{1}=\mathbf{K}_{1}\left[\dot{\mathbf{G}}(\tilde{\mathbf{q}}) \mathbf{z}_{1}+\mathbf{G}(\tilde{\mathbf{q}}) \dot{\mathbf{z}}_{1}\right]
$$

where $\dot{\mathbf{z}}_{1}$ can be found from (40). Moreover, $\dot{\mathbf{G}}(\tilde{\mathbf{q}})$ can be expressed as

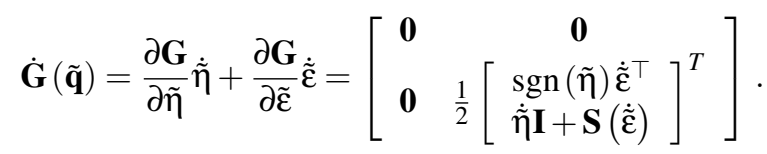

The expressions for $\dot{\tilde{\eta}}$ and $\dot{\tilde{\varepsilon}}$ in (39) can now be inserted to remove the time differentiated states in the controller.

\section{SIMULATIONS}

In this section, simulation results for a leader-follower spacecraft formation are presented to illustrate the performance of the presented control law. In the first three simulations, the orbital perturbations are assumed to be perfectly known or estimated based on measurements. In the fourth simulation, orbital perturbations are uncompensated. In all simulations, we have included aerodynamic drag and $J_{2}$ gravity perturbations based on standard models presented in e.g. [23], [24]. Both the leader and the follower spacecraft have masses $100 \mathrm{~kg}$, and moments of inertia given as $\mathbf{I}=$ $\operatorname{diag}\left\{\begin{array}{lll}4.350 & 4.3370 & 3.6640\end{array}\right\} \mathrm{kgm}^{2}$. The leader spacecraft is assumed to follow an equatorial orbit with a perigee altitude of $250 \mathrm{~km}$ and eccentricity $e=0.3$, and the leader body and orbit coordinate frames are perfectly aligned at all times. The follower spacecraft is assumed to have available
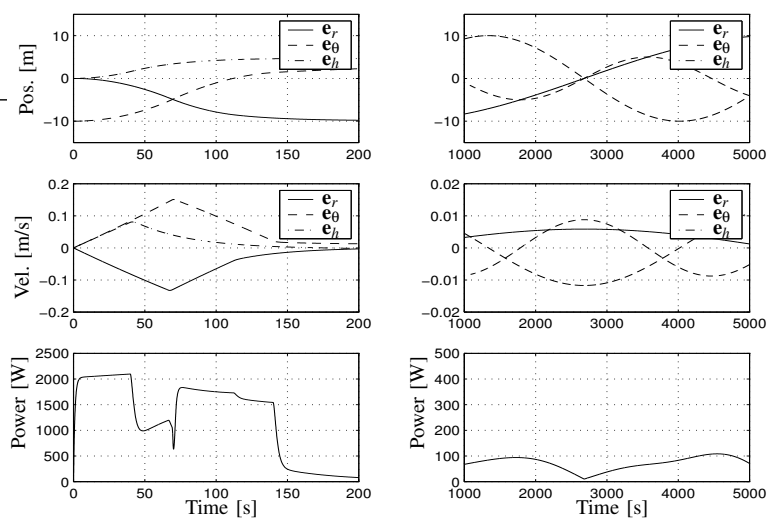

Fig. 2. Relative position and velocity
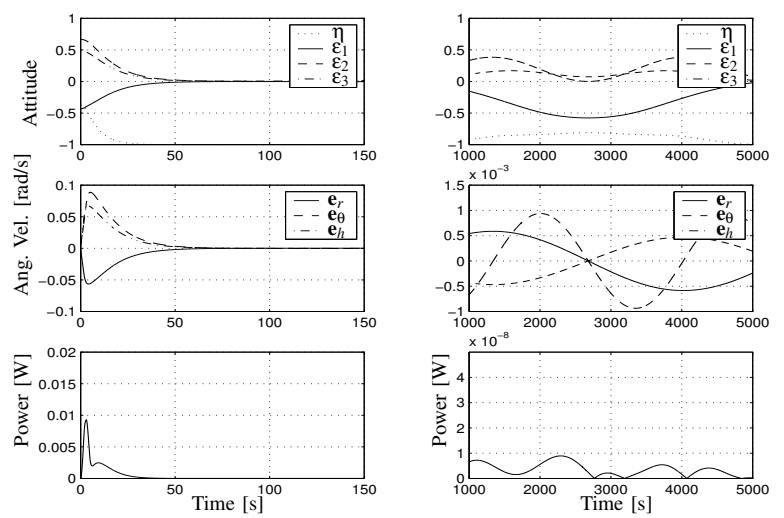

Fig. 3. Relative attitude and angular velocity

continuous actuation in/about all body axes, with a maximum force and torque of $0.2 \mathrm{~N}$ and $0.1 \mathrm{Nm}$, respectively, and the controller gains $\mathbf{K}_{1}=\operatorname{diag}\{0.5,0.5,0.5,0.05,0.05,0.05\}$ and $\mathbf{K}_{2}=\operatorname{diag}\{1,1,1,4,4,4\}$ have been used.

The initial relative positions and attitudes are standstill at $[0,-10,0] \mathrm{m}$ and $\left[-75^{\circ},-175^{\circ}, 70^{\circ}\right]$, respectively. The latter corresponds to the quaternion values $[-0.3772$, $0.4329,0.6645,0.4783]$. The follower spacecraft is commanded to follow smooth sinusoidal trajectories around the origin with velocity and angular velocity profiles

$$
\begin{aligned}
& \mathbf{v}_{*}(t)=\left[\begin{array}{lll}
10 c_{o} \sin \left(c_{o} t\right), & 20 c_{o} \cos \left(2 c_{o} t\right), & -15 c_{o} \sin \left(3 c_{o} t\right)
\end{array}\right]^{\top} \\
& \omega_{*}(t)=\left[\begin{array}{lll}
-c_{o} \sin \left(2 c_{o} t\right), & \frac{8}{5} c_{o} \sin \left(4 c_{o} t\right), \frac{4}{5} c_{o} \sin \left(2 c_{o} t\right)
\end{array}\right]^{\top}
\end{aligned}
$$

where $c_{o}=\frac{\pi}{T_{o}}$ is a leader orbital period constant. A possible scenario for this motion is in-orbit inspection, where the follower moves in orbit around the leader.

\section{A. Results}

The simulation results for relative translation and rotation in the spacecraft formation are presented in Figs. 2 and 3, respectively, and the corresponding error plots are presented in Fig. 4. As shown, both relative translation and rotation converge asymptotically to the reference, and proceed to track the desired trajectories. Note especially that the relative attitude converges to the negative quaternion $-\mathbf{q}_{d}$, which 

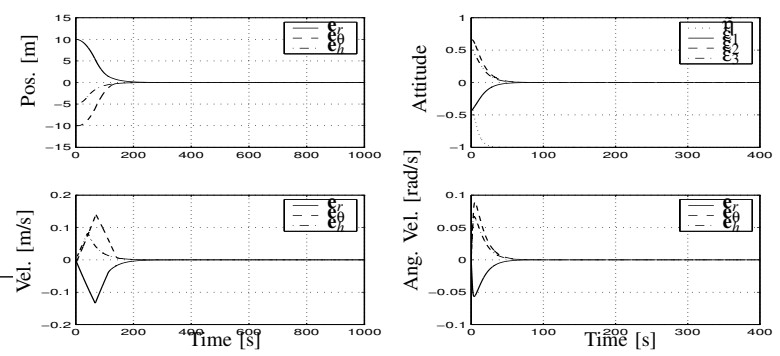

Fig. 4. Position and velocity errors (left), and attitude and angular velocity errors (right)

from our definition in (38) is a valid equilibrium point. So based on the initial and desired attitude, the controller chooses the negative of the desired quaternion as its reference point, since this corresponds to the shortest rotation path. This is a result of our choice of backstepping variables, as mentioned in Remark 1. Fig. 5 shows the state errors
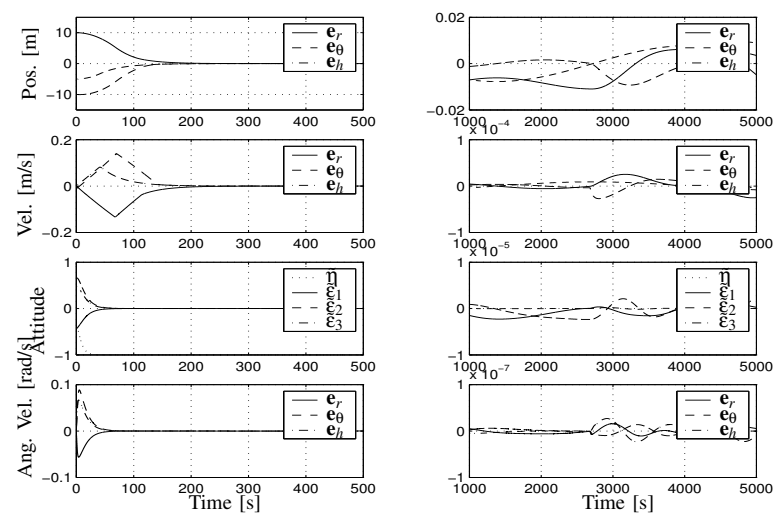

Fig. 5. State errors with uncompensated perturbations

when orbital perturbations are left uncompensated, and the practical stability property mentioned in Remark 3 is easily detected. However, note that even though we do not compensate for aerodynamic drag and $J_{2}$ gravity perturbations, the position and attitude errors are still kept within $0.01 \mathrm{~m}$ and $0.01^{\circ}$, respectively.

\section{CONCLUSION}

In this paper we have presented a $6 \mathrm{DOF}$ Euler-Lagrange model formulation of relative translation and rotation in a leader-follower spacecraft formation. To show the applicability of the model, we also presented a solution to the problem of relative translational and rotational tracking, using the concept of vectorial integrator backstepping. The controller solution presented utilizes the quaternion representation to achieve a shorter rotation path on commanded attitude changes, and the equilibrium points in the closedloop system were proved to be uniformly asymptotically stable. Finally, simulation results were presented the illustrate the performance of the derived control law.

\section{REFERENCES}

[1] G. W. Hill, "Researches in the lunar theory," American Journal of Mathematics, vol. 1, no. 1, pp. 5-26, 1878.
[2] W. H. Clohessy and R. S. Wiltshire, "Terminal guidance system for satellite rendezvous," Journal of Aerospace Sciences, vol. 27, no. 9, pp. 653-658, 1960.

[3] C. R. McInnes, "Autonomous ring formation for a planar constellation of satellites," AIAA Journal of Guidance, Control and Dynamics, vol. 18, no. 5, pp. 1215-1217, 1995.

[4] P. K. C. Wang and F. Y. Hadaegh, "Coordination and control of multiple microspacecraft moving in formation," Journal of the Astronautical Sciences, vol. 44, no. 3, pp. 315-355, 1996.

[5] V. Manikonda, P. O. Arambel, M. Gopinathan, R. K. Mehra, and F. Y. Hadaegh, "A model predictive control-based approach for spacecraft formation keeping and attitude control," in Proc. of the American Control Conf., San Diego, CA, 1999.

[6] Q. Yan, G. Yang, V. Kapila, and M. de Queiroz, "Nonlinear dynamics and adaptive control of multiple spacecraft in periodic relative orbits," in Proc. of the AAS Guidance and Control Conf., Breckenridge, CO, 2000.

[7] H. Pan and V. Kapila, "Adaptive nonlinear control for spacecraft formation flying with coupled translational and attitude dynamics," in Proc. of the Conf. on Decision and Control, Orlando, FL, 2001.

[8] P. K. C. Wang, F. Y. Hadaegh, and K. Lau, "Synchronized formation rotation and attitude control of multiple free-flying spacecraft," AIAA J. of Guidance, Control and Dynamics, vol. 22, no. 1, pp. 28-35, 1999.

[9] H. Wong, H. Pan, and V. Kapila, "Output feedback control for spacecraft formation flying with coupled translation and attitude dynamics," in Proc. of the American Control Conf., Portland, OR, 2005.

[10] R. Ortega, A. Loría, P. J. Nicklasson, and H. Sira-Ramírez, Passivitybased Control of Euler-Lagrange Systems. London: Springer, 1998.

[11] O. Egeland and J. T. Gravdahl, Modeling and Simulation for Automatic Control. Trondheim, Norway: Marine Cybernetics, 2002.

[12] R. Kristiansen, P. J. Nicklasson, and J. T. Gravdahl, "Quaternion-based backstepping control of relative attitude in a spacecraft formation," in Proc. of the 45th IEEE Conf. on Decision and Control, San Diego, CA, 2006.

[13] H. Schaub and J. L. Junkins, Analytical Mechanics of Space Systems, ser. AIAA Education Series. Reston, VA: American Institute of Aeronautics and Astronautics, 2003.

[14] R. H. Battin, An Introduction to the Mathematics and Methods of Astrodynamics, Revised Edition, ser. AIAA Education Series. Reston, VA: American Institute of Aeronautics and Astronautics, 1999.

[15] R. Kristiansen, E. I. Grøtli, P. J. Nicklasson, and J. T. Gravdahl, "A model of relative translation and rotation in leader-follower spacecraft formations," Modeling, Identification and Control, vol. 28, no. 1, pp. 3-14, 2007.

[16] M. J. Sidi, Spacecraft Dynamics and Control. New York: Cambridge University Press, 1997.

[17] O.-E. Fjellstad, "Control of unmanned underwater vehicles in six degrees of freedom," Ph.D. dissertation, Department of Engineering Cybernetics, Norwegian University of Science and Technology, Trondheim, Norway, 1994.

[18] T. I. Fossen, Marine Control Systems: Guidance, Navigation, and Control of Ships, Rigs and Underwater Vehicles. Trondheim, Norway: Marine Cybernetics, 2002.

[19] H. K. Khalil, Nonlinear Systems, third edition. Upper Saddle River, New Jersey, USA: Pearson Education International Inc., 2002.

[20] J. T.-Y. Wen and K. Kreutz-Delgado, "The attitude control problem," IEEE Transactions on Automatic Control, vol. 36, no. 10, pp. 1148$1162,1991$.

[21] S. P. Bhat and D. S. Bernstein, "A topological obstruction to continuous global stabilization of rotational motion and the unwinding phenomenon," Systems \& Control Letters, vol. 39, pp. 63-70, 2000.

[22] R. Kristiansen, A. Loría, A. Chaillet, and P. J. Nicklasson, "Output feedback control of relative translation in a leader-follower spacecraft formation," in Group Coordination and Cooperative Control, ser. Lecture Notes in Control and Information Sciences, K. Y. Pettersen, J. T. Gravdahl, and H. Nijmeijer, Eds. Springer Verlag, 2006, vol. 336, pp. 131-151.

[23] J. R. Wertz, Ed., Spacecraft Attitude Determination and Control. London: Kluwer Academic Publishers, 1978.

[24] O. Montenbruck and E. Gill, Satellite Orbits. Models, methods, applications. Berlin, Germany: Springer-Verlag, 2001, first edition, corrected second printing. 